\title{
El valor agregado de la educación superior en la formación en segunda lengua en Colombia ${ }^{1}$
}

\author{
The added value of higher education in second language \\ training in Colombia
}

Recibido: 23 de julio de 2015- Revisado: 11 de noviembre de 2015 - Aceptado: 09 de diciembre de 2015

Ramiro Rodríguez Revilla ${ }^{2}$

Diana Carolina López Cuevas ${ }^{3}$

\section{Resumen}

En este documento se presenta un modelo para medir el valor agregado de la educación superior en Colombia, discriminado por áreas de conocimiento de los programas académicos. Este es utilizado para evaluar la calidad del sistema educativo en este nivel, específicamente en la competencia del idioma inglés; teniendo como marco normativo el Programa Nacional de Bilingüismo 2004-2019 y el Marco Común Europeo de Referencia. Para llevar a cabo esta medición se emplea una estimación de un modelo lineal jerárquico con variables instrumentales a partir de micro datos suministrados por el Instituto Colombiano para la Evaluación de la Educación y el Departamento Administrativo Nacional de Estadística. El resultado del modelo muestra que los programas de economía, contaduría y administración de empresas son los que más aportan a la formación en inglés en Colombia.

\section{Palabras clave}

Calidad de la educación, modelos de valor agregado, bilingüismo.

\begin{abstract}
This paper presents a model to measure the added value of higher education in Colombia, discriminated by areas of knowledge of academic programs. This is used to assess the quality of the education system at this level, specifically in the english language proficiency; having as a regulatory framework the Programa Nacional de Bilingüismo 2004-2019 and the Marco Común Europeo de Referencia. To carry out this measurement an estimate of a hierarchical linear model is used with instrumental variables from micro data supplied by the Instituto Colombiano para la Evaluación de la Educación (ICFES) and the Departamento Administrativo Nacional de Estadística (DANE). The result of the model shows that the programs of economics, accounting and business administration are the major contributors to training in english in Colombia.
\end{abstract}

\section{Keywords}

Education quality, added value models, bilingualism.

\footnotetext{
${ }^{1}$ El artículo ha sido desarrollado en el marco del proyecto de investigación "Modelo de valor agregado para la educación superior en Colombia", financiado por la Fundación Universitaria Los Libertadores, Bogotá, Colombia.

${ }^{2}$ Economista, docente e investigador de la Fundación Universitaria Los Libertadores, Bogotá, Colombia. Magíster en Ciencias Económicas de la Universidad Santo Tomás, Bogotá, Colombia.

Correo electrónico: rrodriguezre@libertadores.edu.co

${ }^{3}$ Economista e investigadora. Especialista en Formulación de Proyectos del Colegio Mayor de Nuestra Señora del Rosario, Bogotá, Colombia. Correo electrónico: diana.lopez.8701@gmail.com

Para citar este artículo use: Rodríguez, R., López, D. (2016). El valor agregado de la educación superior en la formación en segunda lengua en Colombia. Revista Civilizar Ciencias Sociales y Humanas, 16(30), 119-136.
} 


\section{Introducción}

El Programa Nacional de Bilingüismo (PNB) 2004-2019 establece al idioma inglés como la lengua extranjera a fortalecer, estrategia para el logro de una mayor competitividad internacional que permita a Colombia un crecimiento y desarrollo económico en el largo plazo. En el marco de la Ley General de Educación de 1994', el Estado dispone que todos las instituciones educativas ofrezcan a sus estudiantes la formación en una lengua extranjera desde un nivel básico. El Ministerio de Educación Nacional (MEN) diseñó desde ese entonces políticas y estrategias para fortalecer el dominio del inglés en todos los niveles de educación.

En el año 2004 el MEN adoptó el Marco Común Europeo de Referencia ${ }^{2}$ por la solidez de su desarrollo y aplicabilidad al sistema educativo nacional, para la enseñanza, aprendizaje y evaluación del idioma inglés. Hasta junio de 2009, un grupo compuesto por 78 secretarías de educación hizo una prueba diagnóstica entre los docentes oficiales del nivel básico y medio, encontrando que solo el $10 \%$ de ellos alcanzaba el nivel intermedio B2 o superior. A partir de esta línea base del diagnóstico, las estrategias de acción fijadas por el MEN consisten en la creación y difusión de estándares de inglés para la educación básica y media, delimitación de un sistema de evaluación sólido y coherente y definición y desarrollo de planes de capacitación.

El desempeño de la competencia en inglés en Colombia no tiene los mejores resultados en el ámbito internacional. El ranking mundial más grande de dominio del inglés calculado por Education First (EF) (2014) muestra que Colombia mejora su puntuación en el periodo 2011-2014, aunque esta no representa avances en la clasificación global. Tal es así, que ocupaba el puesto 41 en el año 2011 y ocupa el puesto 42 el año 2014. En el dominio interno, considerando que el PNB determina como meta que al 2019 todos los estudiantes de educación media tengan un nivel B1 y los que terminan la educación superior un nivel B2 (MEN, 2006), se evidencia que en el nivel medio solo el $13 \%$ de los estudiantes que presentaron la prueba Saber 11 en el periodo 2007-2014 y a nivel superior el $25 \%$ de los estudiantes que presentaron las pruebas Ecaes o Saber Pro en el periodo 20072013, tienen un nivel intermedio B1 o superior.

La evaluación de estas metas en la educación superior con base en promedios simples de los resultados de las pruebas Ecaes y Saber Pro en el módulo de inglés, sería sesgada debido a que no aísla los conocimientos previos de los estudiantes ni factores familiares o socioeconómicos, ya que estudiantes con un desempeño académico notable en su educación básica y media tienen una alta probabilidad de continuar con un elevado rendimiento en niveles superiores independientemente de la institución a la que asisten.

Para evitar este sesgo en las mediciones, es necesario aprovechar los modelos de valor agregado con el objetivo de encontrar el efecto escuela, entendido como aquellas actividades, procesos o estrategias de enseñanza-aprendizaje que brindan las instituciones educativas para contribuir al desarrollo cognitivo de sus estudiantes (McCaffrey, Lockwood, Koretz, Louis, y Hamilton, 2004; Meyer, 1997; Raudenbush, S., 2004; Hanushek, Kain, Rivkin, \& Branch, 2005; Sanders, 2000).

Determinar la calidad de la enseñanza de educación superior midiendo el valor agregado aportado por la institución, aislando los factores sociales y económicos y los conocimientos previos de los estudiantes así como la formación académica de los docentes; es un aporte a la construcción de calidad en el país. En el contexto latinoamericano, Chile tiene desde el año 2012 una propuesta de formulación de política pública, en la cual se recomienda el manejo de modelos de valor agregado para medir el aporte de instituciones escolares a la formación académica de niños y jóvenes. De acuerdo con San Martín y Carrasco (2012), 
estas estrategias permitirán una mejora en las relaciones Estado, familias y escuelas.

Para Colombia, los estudios que reportan el valor agregado de la educación son pocos. Bogoya y Bogoya (2013) proponen un modelo matemático lineal que representa el valor académico agregado en Colombia para estudiantes de administración considerando 10.566 alumnos correspondientes a los resultados de la prueba Saber Pro en el año 2009. Ellos encuentran que los conocimientos anteriores medidos por la prueba Saber 11 explican 13 veces más la varianza de los resultados de la prueba Saber Pro que el estrato socioeconómico de los estudiantes.

Por su parte, la Organización para la Cooperación y el Desarrollo Económicos (2013) ha tomado a Colombia como uno de los países modelo para aplicar el piloto de la prueba Assessment of Higher Education Learning Outcomes (AHELO). Esta prueba medirá el valor agregado de la educación superior en un contexto internacional. El Instituto Colombiano para la Evaluación de la Educación (ICFES, por su antigua sigla de: Instituto Colombiano para el Fomento de la Educación Superior) ha adelantado estudios de valor agregado para la educación superior sin una publicación oficial de los resultados.

En este sentido, el objetivo de esta investigación radica en medir el valor agregado o efecto escuela de las instituciones de educación superior en el ámbito de pregrado en la formación en segunda lengua. El modelo tomará como insumo los resultados de las evaluaciones Saber 11, Ecaes y Saber Pro; la primera se utiliza para incorporar al modelo el desempeño de los conocimientos aprendidos por los estudiantes al inicio de la educación terciaria y las segundas, los logros finalizando esta etapa; de tal manera que se considera dos evaluaciones por cada estudiante en el tiempo.

Para realizar la medición se construyó una base de datos tipo panel que contiene in- formación para $194.865^{3}$ estudiantes que rindieron la prueba Saber 11 entre los años 2002 y 2008 y la prueba Saber Pro en el periodo 20072012, a partir de microdatos publicados por el ICFES. Además, se agregó a este panel de datos información correspondiente a docentes de instituciones de educación superior en el periodo 2007-2012, extraída del Departamento Administrativo Nacional de Estadística (DANE). Metodológicamente, se controlará por las características familiares, sociales y económicas de cada uno de los estudiantes e institucionales de cada universidad, como son la cantidad de profesores según su formación académica o el carácter de pública o privada.

Esta investigación es un aporte a la construcción de parámetros de medida de la calidad de la enseñanza superior, con el propósito de que en el mediano plazo las mejores prácticas y estrategias de enseñanza-aprendizaje aplicadas por instituciones colombianas sean divulgadas, y de alguna manera sean replicadas en el resto de instituciones.

Por último, este documento consta de cinco secciones, en la primera se hace una breve revisión teórica y desarrollos conceptuales de la relación entre la asimilación de competencias en inglés y el crecimiento y desarrollo económico de los países, para evidenciar la propuesta teórica del PNB 2004-2019. En la segunda se muestran los resultados de la prueba internacional y los hechos estilizados a partir de la base de datos construida para esta investigación. Luego se expone la estrategia empírica para hallar el valor agregado y la estimación del modelo jerárquico lineal. En la cuarta se analizan los resultados del modelo, proponiendo recomendaciones de política a la luz de lo encontrado en la sección anterior. En la última sección se concluye.

\section{Marco referencial}

El nivel de desempeño de una competencia de lectura y escritura en una segunda lengua puede ser visto como una forma de acumulación 
de capital humano por medio de la educación. La apuesta teórica del PNB argumenta que una mejor aprehensión de competencias en segunda lengua aporta a una mayor competitividad internacional, crecimiento y desarrollo económico. La literatura reconoce al menos tres canales a través de los cuales la educación puede impactar sobre el crecimiento y desarrollo económico.

Primero, la educación puede incrementar el capital humano de la fuerza laboral y por lo tanto la productividad (Lucas, 1989, Mankiw, Romer, \& Weil, 1992). Segundo, la educación puede aumentar la capacidad de innovación de la economía impulsando el sector de investigación y desarrollo que genera productos con valor agregado impactando el sector productivo (Romer, 1990). Y por último, puede facilitar la difusión y transmisión de conocimiento necesario para procesar las nuevas tecnologías (Benhabib \& Spiegel, 1994).

Como evidencia empírica Lee (2009) encontró que países con un mejor desempeño en una competencia de segunda lengua medida por el resultado del test TOEFL ${ }^{4}$ crecen a una tasa más acelerada. El efecto del idioma sobre el crecimiento del producto también lo demuestran Hall y Jones (1999), quienes atribuyen las diferencias en el producto por persona en el ámbito internacional al grado de infraestructura social y este se explica parcialmente por la proporción de la población que habla uno de los cinco idiomas básicos de Europa occidental: inglés, francés, alemán, portugués y español.

Teóricos del capital humano y del desarrollo argumentan que la formación en inglés es la clave del éxito de las estrategias orientadas hacia la exportación en el este asiático (Birdsall, Ross, \& Sabot, 1997; McMahon, 1999). En estos países la demanda de mano de obra calificada con competencias en inglés es mayor que en aquellos que los que impulsan una estrategia comercial hacia adentro. La brecha salarial entre trabajadores calificados en un segundo idioma y no calificados genera una sobredemanda por educación que debe ser cubierta por instituciones públicas y privadas. El predominio del idioma inglés como lenguaje de los negocios tiene efectos positivos para el intercambio comercial de las naciones, según las teorías del comercio gravitacional (Helliwell, 1999).

Por otro lado, es ampliamente aceptado que la mayor parte de nuevo conocimiento es generado en países desarrollados, en los cuales el inglés tiene una posición importante en la cultura de las personas. Crystal (2003) ofrece una vasta discusión de la influencia del idioma inglés y evidencia su impacto sobre la sociedad moderna. De esta manera la hegemonía de la lengua inglesa es percibida en distintos aspectos, por ejemplo Graddol (2006) establece que el $53 \%$ de los estudiantes internacionales recibe clases en este lenguaje.

La mejor prueba que soporta la importancia de la aprehensión de competencias en inglés como sinónimo de éxito viene del mercado laboral. Existe una brecha salarial positiva entre las personas que han asimilado la competencia en un segundo idioma frente a los que carecen de esta habilidad (Chiswick, Patrinos, y Hurst, 2000; Bleakey y Chin, 2004).

Otro motivo por el cual el aprendizaje de un segundo idioma es importante, radica en que las empresas multinacionales desean mano de obra bilingüe. Marschan, Welch y Welch (1997) argumentan que las firmas japonesas prefieren invertir en países con altos niveles de competencia bilingüe de sus trabajadores, con las consecuentes remuneraciones más altas que el promedio de empresas domésticas. El impacto del conocimiento del idioma inglés en las actividades laborales diarias se podría describir inicialmente por el hecho de que los manuales de operación y técnicos preventivos y correctivos de las máquinas industriales, así como del software desarrollado con tecnología de frontera vienen en este idioma. Para Keller (2002) las competencias en inglés son primordiales en la difusión de tecnologías. 


\section{Hechos estilizados}

En esta sección se describirá el desempeño del aprendizaje del idioma inglés de personas colombianas mayores de 18 años en el ámbito internacional empleando la prueba practicada por EF. Así como el resultado de las pruebas Saber 11, Ecaes y Saber Pro en el periodo 2007-2014, en el módulo de competencias genéricas correspondientes al inglés, las cuales se encargan de evaluar la educación media y superior en Colombia.
La cuarta edición del test del índice del nivel de inglés (EPI, english proficiency index) perteneciente a $\mathrm{EF}$, consideró a 63 países que no tienen como idioma oficial al inglés para practicar la prueba a más de 750000 personas mayores de 18 años. Estos son evaluados en secciones de gramática, vocabulario, lectura y comprensión. En la gráfica 1 se puede apreciar la correlación directa entre el producto per cápita de los 63 países y el puntaje obtenido en el test. Es decir, a mayor capacidad adquisitiva de los habitantes mejor es el desempeño en la prueba.

Gráfica 1

Correlación entre el puntaje english proficiency index y el producto per cápita 2013 en dólares a precios corrientes

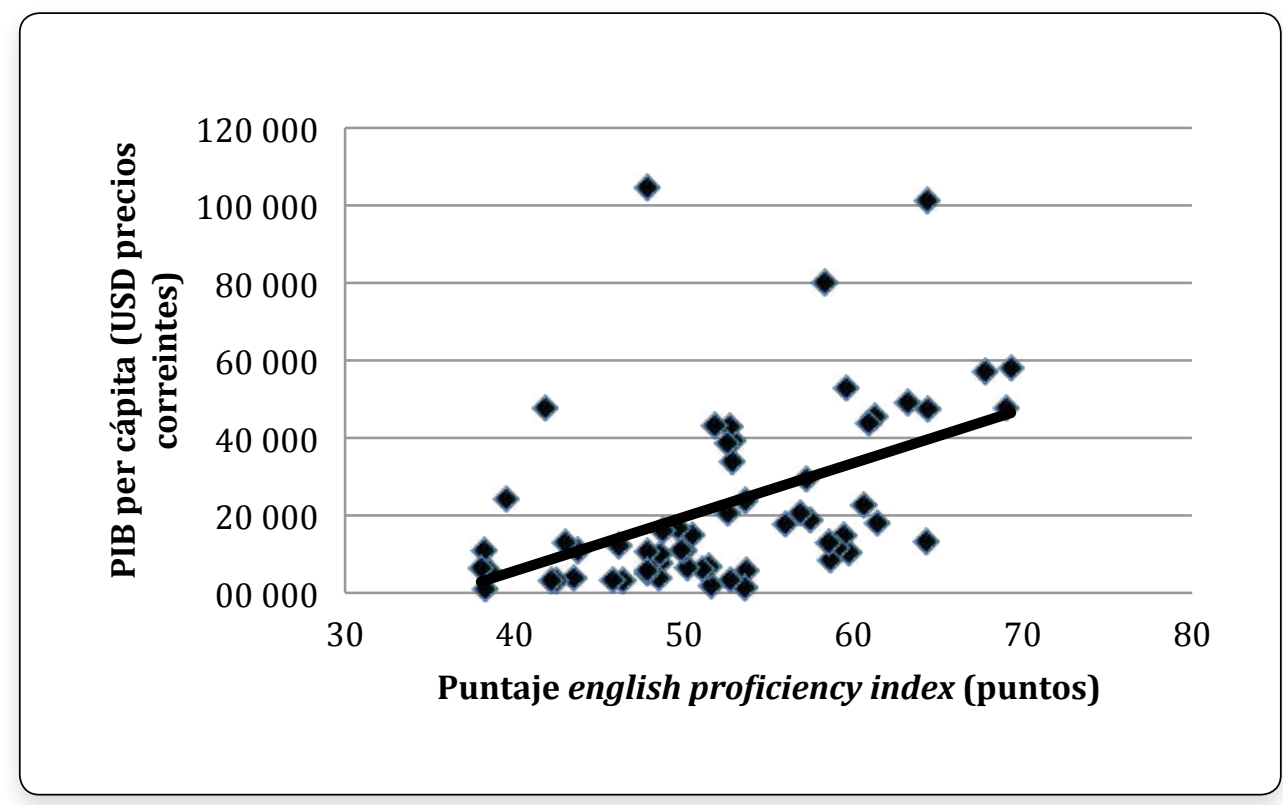

Fuente: elaboración propia con base en datos de EF, 2014 y el Fondo Monetario Internacional, 2016

En la gráfica 2 se puede observar la clasificación de los países de Latinoamérica en la prueba del 2014. Argentina, República Dominicana y Perú tienen los mejores desempeños de la región. La Ley Nacional de Educación del 2006 en Argentina dispuso la enseñanza obligatoria de inglés en todos los colegios oficiales desde el cuarto al doceavo grado. Por su parte, República Dominicana es la nación del hemisferio con más avances en el aprendizaje de in- glés, evidenciándose que en el 2013 existieron más de cien empresas de atención telefónica en inglés empleando a más de 35.000 trabajadores (EF, 2014). En el caso del Perú es notable la cantidad de profesores dedicados a la enseñanza en todos los niveles educativos, en efecto, este país dispone de una de las razones más bajas en la proporción de cantidad de estudiantes por docente, la cual asciende a 21 (Instituto Nacional de Estadística e Informática de Perú, 2014). 
Gráfica 2

Clasificación de países de Latinoamérica en el Education First english proficiency index 2014

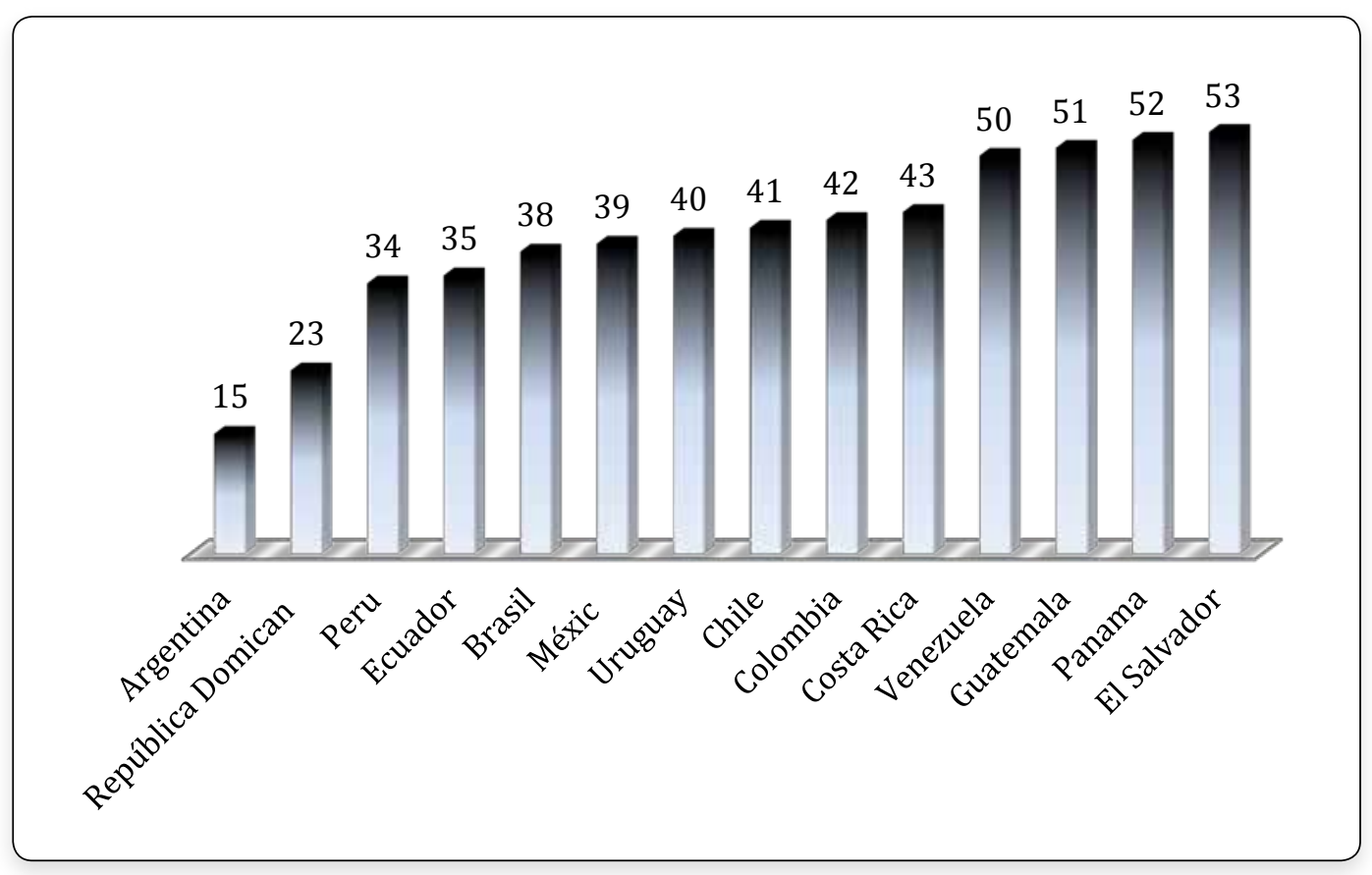

Fuente: elaboración propia con base en datos de EF, 2014

Colombia presenta un comportamiento creciente en el puntaje de la EPI desde el 2011, pero no logra mejorar en la clasificación internacional (véase gráfica 3). Ocupó el puesto 41 en el 2011 y el 42 en el 2014, como se observa en la gráfica 2 .

Gráfica 3

Desempeño de Colombia en el test

Education First english proficiency index 2011-2014

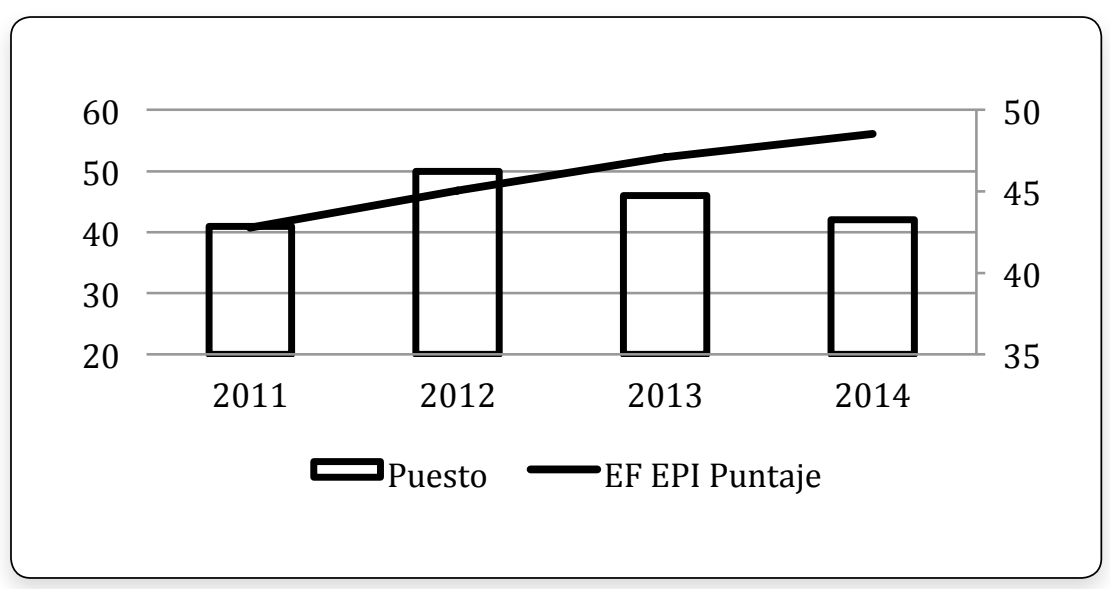

Fuente: elaboración propia con base en datos de EF, 2014 
En el ámbito interno, a partir del año 2007 los componentes de inglés de las pruebas de evaluación llevadas a cabo por el ICFES se encuentran alineados a los estándares y niveles del Marco Común Europeo de Referencia. En la gráfica 4 se observa la relación directa entre el puntaje en inglés obtenido por los estudiantes en la prueba Saber 11 y el obtenido en la prueba
Saber Pro. Se aprecia una fuerte correlación que asciende a 0,78 , es decir, estudiantes que les va mal en su desempeño en la educación media, les va mal en la educación superior. Esto indicaría que se debe fortalecer la enseñanza del segundo idioma desde la infancia y adolescencia, para no depender de lo que se pueda hacer en el nivel superior.

Gráfica 4

Correlación puntajes de inglés de pruebas Saber 11 y Saber Pro, 2002-2012

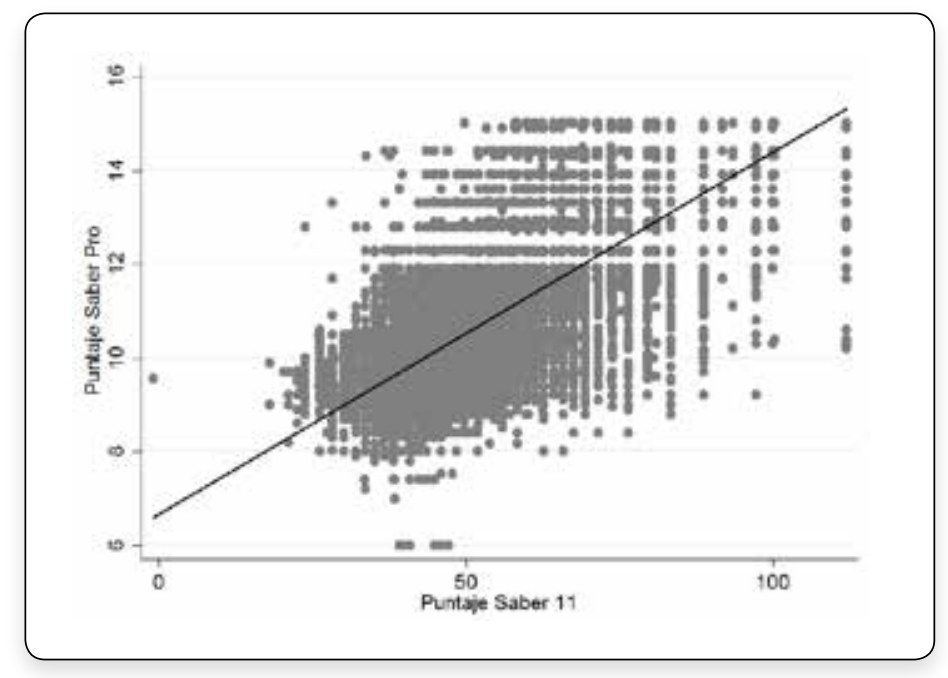

Fuente: elaboración propia con base en datos del ICFES, 2015

En la tabla 1 se reportan las competencias esperadas por los estudiantes según los distintos niveles del Marco Común Europeo de Referencia, utilizados por el MEN en sus distintas metas y evaluaciones. Los niveles A- y B + han sido creados por el MEN, de esta manera: el nivel A- se usa para clasificar a todos los estudiantes que tienen un nivel de inglés muy bajo, inferior al nivel más bajo A1 del Marco. Considerando que hay pocos estudiantes por encima del nivel B1, el MEN los agrupa en $\mathrm{B}+$.

Tabla 1

Niveles y competencias según el Marco Común Europeo de Referencia utilizados por el Ministerio de Educación Nacional

\begin{tabular}{|l|l|l|}
\hline Categoría & Nivel & Competencias \\
\hline B+ & Supera al nivel B1 \\
\hline Intermedio & B1 & $\begin{array}{l}\text { Es capaz de comprender los puntos principales de textos claros y en lengua estándar. Sabe desen- } \\
\text { volverse en la mayor parte de las situaciones que pueden surgir durante un viaje por zonas donde } \\
\text { se utiliza la lengua. Es capaz de producir textos sencillos y coherentes. Puede describir expe- } \\
\text { riencias, acontecimientos, deseos y aspiraciones, así como justificar brevemente sus opiniones o } \\
\text { explicar sus planes }\end{array}$ \\
\hline
\end{tabular}




\begin{tabular}{|c|c|c|}
\hline Categoría & Nivel & Competencias \\
\hline \multirow{3}{*}{ Básico } & A2 & $\begin{array}{l}\text { Es capaz de comprender frases y expresiones de uso frecuente relacionadas con áreas de expe- } \\
\text { riencia que le son especialmente relevantes. Sabe comunicarse a la hora de llevar a cabo tareas } \\
\text { simples y cotidianas que no requieran más que intercambios sencillos y directos. Sabe describir } \\
\text { en términos sencillos aspectos de su pasado y su entorno así como cuestiones que atañen a sus } \\
\text { necesidades inmediatas }\end{array}$ \\
\hline & A1 & $\begin{array}{l}\text { Es capaz de comprender y utilizar expresiones cotidianas de uso muy frecuente así como frases } \\
\text { sencillas destinadas a satisfacer necesidades de tipo inmediato. Puede presentarse a sí mismo y a } \\
\text { otros, pedir y dar información personal básica sobre su domicilio, sus pertenencias y las personas } \\
\text { que conoce. Puede relacionarse de forma elemental siempre que su interlocutor hable despacio y } \\
\text { con claridad y esté dispuesto a cooperar }\end{array}$ \\
\hline & A- & No alcanza el nivel A1 \\
\hline
\end{tabular}

Fuente: elaboración propia con base en información del MEN, 2006

Considerando los niveles del Marco Común Europeo de Referencia, el MEN en su PNB estableció metas para el 2019 en el desempeño de los estudiantes egresados de educación media y superior. De acuerdo con esto, el MEN (2006) propuso que los estudiantes de educación media alcanzaran la categoría B1 y los de educación superior la B2.

En la gráfica 5 se muestra la evolución del desempeño de inglés de los estudiantes de educación media evaluados en la prueba Saber 11 en el periodo 2007-2014. Se puede apreciar que en el 2007 solo el $5 \%$ de los estudiantes tenía un nivel B1 o superior, y este segmento representa el $19 \%$ en el 2014. Haciendo un corte en el 2014, se evidencia que en un poco más de la mitad del tiempo de ejecución del PNB aún el $81 \%$ de los egresados de educación media no ha superado la meta propuesta (véase tabla 2).

\section{Gráfica 5}

Evolución del desempeño en inglés de los estudiantes de educación media, Saber 11, 2007-2014

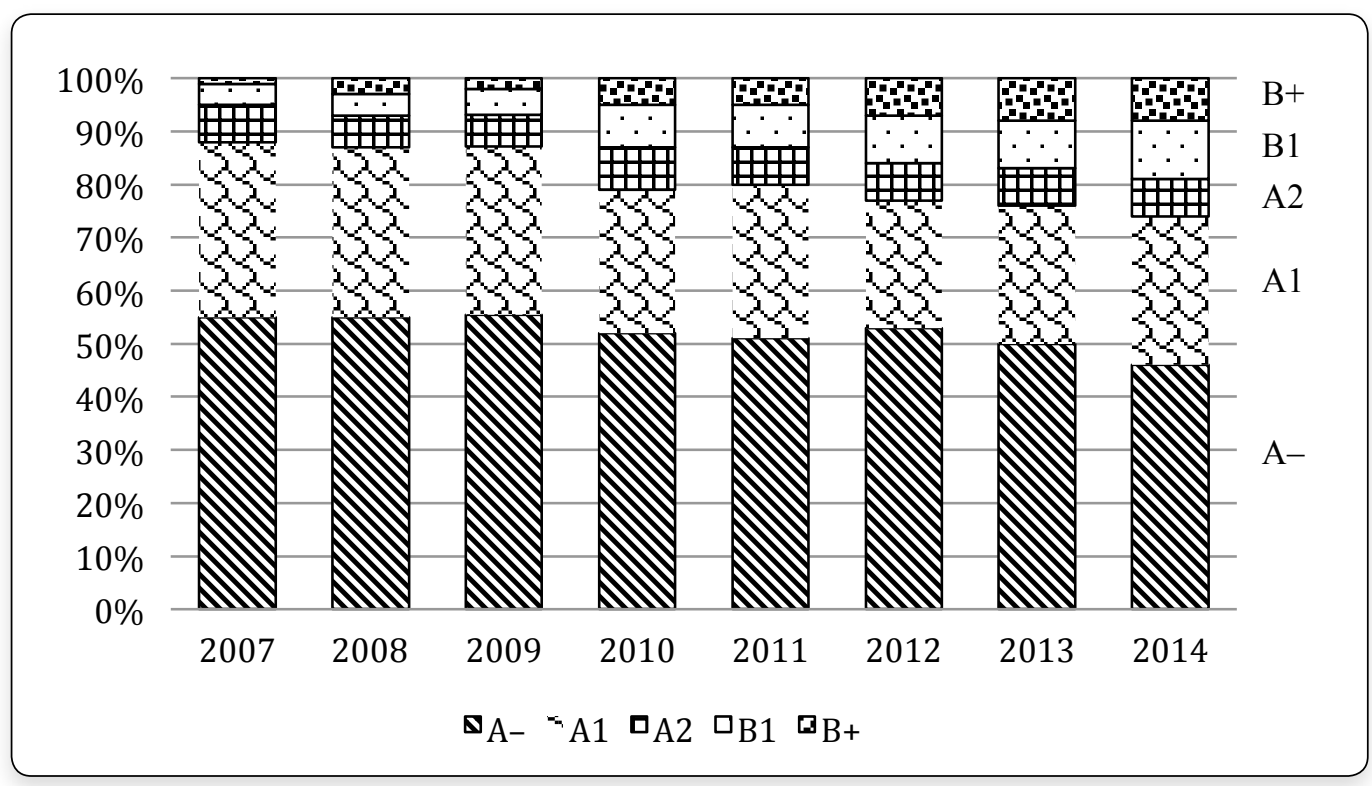

Fuente: elaboración propia con base en información del ICFES, 2015 
El panorama para los egresados de la educación superior es más desalentador que para los bachilleres de la educación media. En el año 2007, solo el 7 \% de los estudiantes de educación superior tenía una competencia categorizada en un nivel superior a B1 y en el 2013 , este segmento representa el $10 \%$ (véase gráfica 6).

\section{Gráfica 6}

Evolución del desempeño en inglés de los estudiantes de educación superior, Ecaes/Saber Pro, 2007-2013

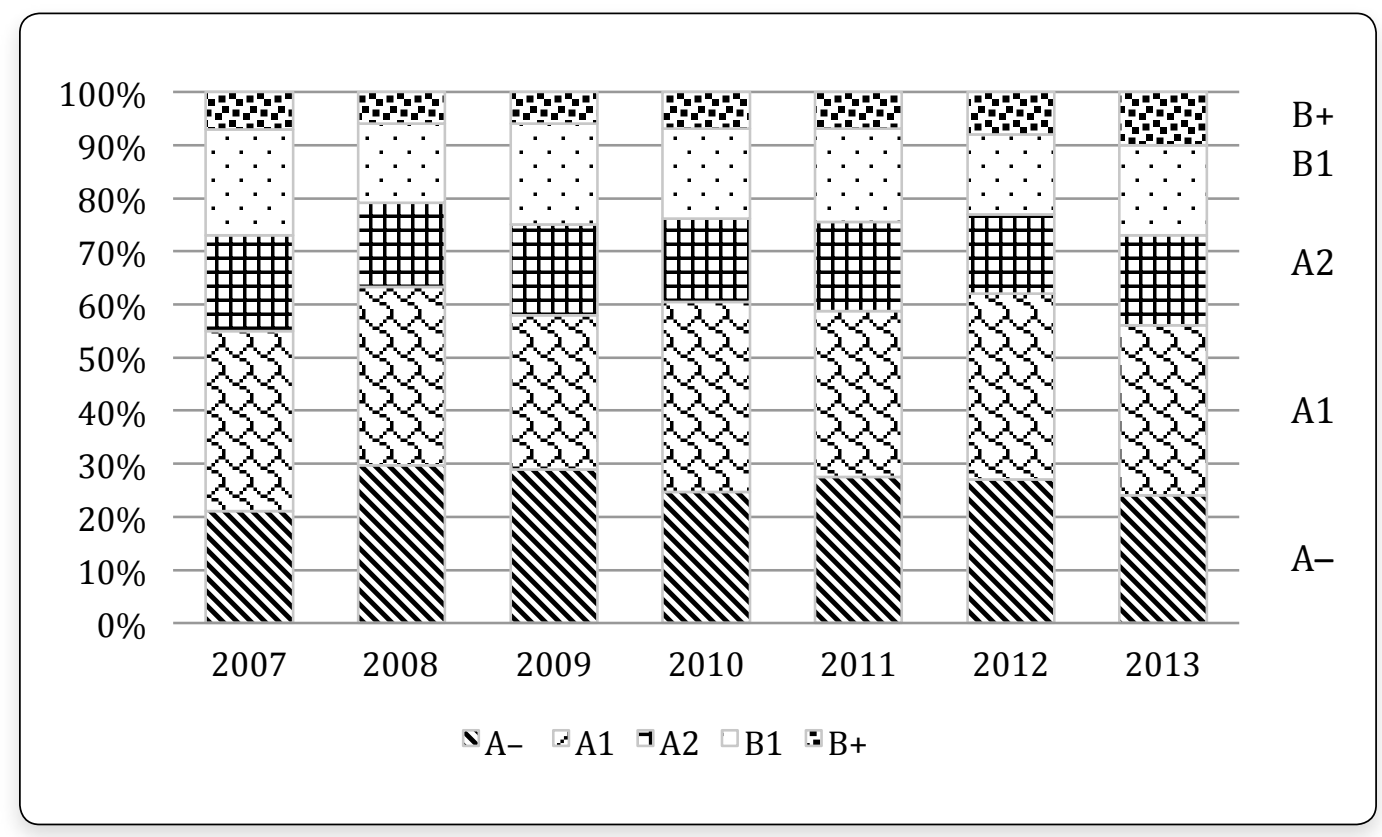

Fuente: elaboración propia con base en información del ICFES, 2015

En la tabla 2 se advierte que las metas propuestas por el PNB en el año 2004 muy difícilmente se lograrán. Con las dos terceras partes del periodo de ejecución del PNB cumplidos, el 81 $\%$ de los estudiantes de educación media está por debajo del nivel B1 y el $90 \%$ de los estudiantes de educación superior tiene un nivel de inglés inferior a la meta B2. En efecto, el avance en el periodo 2007-2013/2014 ha sido mínimo, $14 \%$ para el nivel medio y $3 \%$ para el superior.

Tabla 2

Estado actual (2013/2014) y metas (2019) del aprendizaje de inglés de los estudiantes colombianos de educación media y superior

\begin{tabular}{|ccccc|}
$\begin{array}{c}\text { Nivel } \\
\text { educativo }\end{array}$ & $\begin{array}{c}\text { Meta } \\
\text { competencia }\end{array}$ & $\begin{array}{c}\mathbf{2 0 0 7} \\
(\%)\end{array}$ & $\begin{array}{c}\text { 2013/2014 } \\
(\%)\end{array}$ & $\begin{array}{c}\text { Meta al } 2019 \\
(\%)\end{array}$ \\
\hline Educación media & B1 & 5 & $19 *$ & 100 \\
\hline Educación superior & B2 & 7 & $10^{* *}$ & 100
\end{tabular}

Nota: * al 2014, ** al 2013.

Fuente: elaboración propia con base en información del ICFES, 2015 


\section{Modelo de valor agregado}

Metodológicamente, la variación en el retorno a la escolaridad proviene de muchas fuentes, una de las más importantes analiza la calidad de la educación con base en resultados de pruebas estandarizadas que miden el desempeño académico. Entre las técnicas más adecuadas para medir el logro escolar se encuentran: el uso de variables instrumentales con regresiones discontinuas, estructuras discretas de selección y los modelos jerárquicos lineales (hierarchical linear models, HLM). En Meghir y Rivkin (2011) se puede encontrar una revisión de la literatura acerca de los métodos econométricos para la investigación en educación.

Los modelos que utilizan estructuras jerárquicas fueron introducidos por Lindley y Smith (1972) y Smith (1973) para casos con covarianza simple; pero esta estructura no permitía modelar especificaciones más complejas. Si bien tales modelos se desarrollaron teóricamente en los setenta, fueron Aitkin y Longford (1986) quienes propusieron una técnica de análisis que ha marcado la investigación educativa desde entonces, los modelos multinivel o modelos jerárquico-lineales.

Paralelo a los desarrollos de las estructuras jerárquicas, el sistema educacional en el Reino Unido estableció en 1986 las bases del sistema de rankings o clasificación de escuelas, denominado League Tables (San Martín y Carrasco, 2012). Esta clasificación consideraba los resultados promedio de instituciones de educación secundaria, como indicadores de calidad de esos colegios. Más adelante, Goldstein, Huiqi, Rtah y Hill (2000) demostraron que las metodologías de clasificación empleadas se apoyaban en estimaciones inapropiadas por cuanto consideraban solo la puntuación final de promedios simples sin controlar por variables determinadas anteriormente en el tiempo.

Además Goldstein (2001) demostró que esta clasificación es modificada, corregida y tiene una mejor explicación haciendo uso de los modelos de valor agregado introducidos por Draper (1995), Goldstein y Spiegelhalter (1996), Longford (1993) y Raudenbush y Willms (1995). Estos privilegian en su metodología de cálculo, los modelos multinivel. Inglaterra ha seguido empleando modelos de valor agregado para capturar los determinantes del logro escolar y así divulgar las League Tables.

La base de datos utilizada para elaborar el modelo considera una estructura tipo panel que contiene información para $194.865^{5}$ estudiantes que han rendido la prueba Saber 11 entre los años 2002 y 2008 y la prueba Saber Pro en el periodo 2007-2012, a partir de microdatos publicados por el Icfes. Para medir el conocimiento en segunda lengua, se ha tomado el resultado de inglés, que es una competencia genérica evaluada en la prueba Saber Pro. En la tabla 3 se aprecia las estadísticas descriptivas para el resultado del módulo de inglés, en esta se muestra que las escalas en el periodo no han cambiado, lo que a su vez garantiza la comparabilidad de los resultados. Además, se ha agregado a este panel de datos, información correspondiente a docentes de instituciones de educación superior (como su formación académica, vinculación con la institución y su procedencia nacional o extranjera) en el periodo 2007-2012, extraída del DANE.

Tabla 3

Estadísticas descriptivas para el resultado de inglés de la prueba Saber Pro 2007-2012

\begin{tabular}{|rrrrr|}
\hline Año & Observaciones & Promedio & Valor mínimo & Valor máximo \\
\hline 2007 & 12.443 & 10,5 & 7,1 & 14,1 \\
\hline 2008 & 14.478 & 10,3 & 7,6 & 15,0 \\
\hline 2009 & 40.867 & 10,5 & 6,0 & 14,9 \\
\hline
\end{tabular}




\begin{tabular}{|crccc|}
\hline Año & Observaciones & Promedio & Valor mínimo & Valor máximo \\
\hline 2010 & 42.403 & 10,5 & 6,0 & 15,0 \\
\hline 2011 & 41.603 & 10,5 & 6,0 & 15,0 \\
\hline 2012 & 46.016 & 10,6 & 6,0 & 15,0 \\
\hline
\end{tabular}

Fuente: elaboración propia con base en información del ICFES, 2015

Para medir el valor agregado de la formación en inglés de la educación superior en el ámbito nacional se estimaron modelos lineales jerárquicos discriminados por las ocho áreas de conocimiento según la tabla 4 . Se puede apreciar que las áreas de conocimiento más demandadas son ingeniería, arquitectura, economía, administración y contaduría.

Tabla 4

Áreas de conocimiento de los programas académicos, su codificación y cantidad de observaciones en el modelo

\begin{tabular}{|lcc|}
\hline \multicolumn{1}{c}{ Área de conocimiento } & Codificación en el modelo & Cantidad de observaciones \\
\hline Agronomía y veterinaria & agro & 2.365 \\
\hline Bellas artes & bela & 5.184 \\
\hline Ciencias de la educación & educ & 14.016 \\
\hline Ciencias de la salud & salu & 17.039 \\
\hline Ciencias sociales y humanas & soch & 26.014 \\
\hline Economía, administración y contaduría & ecad & 38.609 \\
\hline Ingeniería y arquitectura & ing & 39.752 \\
\hline Matemáticas y ciencias naturales & matn & 3595 \\
\hline
\end{tabular}

Fuente: elaboración propia con base en información del ICFES, 2015

Se consideraron once variables que actúan como independientes o también denominadas covariables en la literatura de los HLM cuya descripción se muestra en la tabla 5.

Tabla 5

Descripción de las variables y covariables de los modelos

\begin{tabular}{|c|c|c|c|}
\hline & Nombre & Descripción & Valores \\
\hline $\begin{array}{c}\text { Variable } \\
\text { dependiente }\end{array}$ & Ingles_sbpro & $\begin{array}{l}\text { Puntaje obtenido en la prueba de inglés de la } \\
\text { prueba Saber Pro }\end{array}$ & Entre 6,2 y 17,29 \\
\hline \multirow{7}{*}{$\begin{array}{l}\text { Covariables } \\
\text { del primer } \\
\text { nivel }\end{array}$} & Ingles_sb11 & $\begin{array}{l}\text { Puntaje obtenido en la prueba Saber } 11 \text { en el } \\
\text { módulo de inglés }\end{array}$ & \\
\hline & male & Género & 1: hombre, 0 : mujer \\
\hline & Educa_padre & Años de educación del padre & $\begin{array}{l}\text { De } 0 \text { hasta } 17 \text {. Por niveles, } 0 \text { : ningún nivel de } \\
\text { educación, } 17 \text { : posgrado }\end{array}$ \\
\hline & Educa_madre & Años de educación de la madre & $\begin{array}{l}\text { De } 0 \text { hasta } 17 \text {. Por niveles, } 0 \text { : ningún nivel de } \\
\text { educación, } 17 \text { : posgrado }\end{array}$ \\
\hline & Estu_estrato & $\begin{array}{l}\text { Nivel socioeconómico del estudiante representado } \\
\text { por el estrato de la vivienda }\end{array}$ & $\begin{array}{l}1,2,3,4,5 \text { y } 6 \text {. Donde } 6 \text { representa el nivel más } \\
\text { alto }\end{array}$ \\
\hline & Ingfamilia & Ingreso familiar mensual & $\begin{array}{l}\text { De } 1 \text { hasta } 7 \text {. Por niveles de ingreso desde menos } \\
\text { de un salario mínimo hasta más de diez salarios } \\
\text { mínimos mensuales }\end{array}$ \\
\hline & Trabaja & Inclusión laboral del estudiante & 1: trabaja, $0:$ no trabaja \\
\hline
\end{tabular}




\begin{tabular}{|c|l|l|l|}
\hline & \multicolumn{1}{|c|}{ Nombre } & \multicolumn{1}{c|}{ Descripción } & \multicolumn{1}{c|}{ Valores } \\
\hline & Univ_privada & Tipo de universidad & 1: privada, 0: pública \\
\hline $\begin{array}{c}\text { Covariables } \\
\text { del segundo } \\
\text { nivel }\end{array}$ & nmtc & $\begin{array}{l}\text { Tipo de selección de la universidad } \\
\text { Cantidad de docentes nacionales tiempo completo } \\
\text { con maestría de esa universidad }\end{array}$ & $\begin{array}{l}\text { 1: la universidad escoge a los aspirantes, 0: no } \\
\text { escoge }\end{array}$ \\
\hline & nprtc & $\begin{array}{l}\text { Cantidad de docentes nacionales tiempo completo } \\
\text { profesionales de esa universidad }\end{array}$ & \\
\hline
\end{tabular}

Fuente: elaboración propia con base en datos del ICFES, 2015

Para la estimación del modelo se escogió uno cuya especificación es de coeficientes aleatorios, el cual incluye pendientes e interceptos aleatorios. Se sigue la formulación de Raudenbush y Bryk (2002), en la cual se detalla el modelo en dos niveles. El primer modelo corresponde a información perteneciente a los estudiantes (incluyendo resultados de pruebas y variables sociales y económicas) y el segundo cuantifica información relevante para las universidades (tipo de universidad, selección e información de los docentes). La especificación del primer nivel se puede observar en la ecuación (1).

$$
\text { ingles_sbpro } \mathrm{i}_{\mathrm{i}, \mathrm{j}}=\beta_{0, \mathrm{j}}+\beta_{1, \mathrm{j}} x_{\mathrm{i}, \mathrm{j}}+u_{\mathrm{i}, \mathrm{j}}
$$

Donde:

$\beta_{0, \mathrm{j}}$ : intercepto aleatorio específico de cada universidad.

$\beta_{1, \mathrm{j}}$ : pendiente aleatoria específica de cada universidad.

$$
x_{\mathrm{i}, \mathrm{j}} \text { : covariables del primer nivel. }
$$

La especificación del segundo nivel sigue la formulación de la ecuación (2).

$$
\begin{aligned}
& \beta_{0, \mathrm{j}}=\alpha_{0,0}+w_{1, \mathrm{j}}+v_{0, \mathrm{j}} \\
& \beta_{1, \mathrm{j}}=\alpha_{1,0}+\mathrm{w}_{1, \mathrm{j}}+\mathrm{v}_{1, \mathrm{j}}
\end{aligned}
$$

\section{Donde:}

$\alpha_{0,0}$ : media global del desempeño en inglés de toda la muestra. $\mathrm{w}_{1, \mathrm{j}}$ : covariable del segundo nivel, no varía en el primer nivel.

$v_{0, \mathrm{j}}$ : error que mide el valor agregado de la institución, cuando la media $\alpha_{0,0}$ y la covariable $w_{1, \mathrm{j}}$ son ceros.

Los estimadores de efectos aleatorios $\beta_{0, \mathrm{j}}$ y $\beta_{1, \mathrm{j}}$ no son observados, se debe sustituir (2) en (1) para obtener las formas reducidas (Rabe-Hesketh \& Skrondal, 2012).

Considerar a la variable que representa el desempeño anterior de cada estudiante, en este caso el resultado de la prueba de lenguaje del Saber 11, como una covariable del modelo a estimar, representaría estimadores sesgados (Manzi, San Martin \& Van Bellegem, 2012). Para solucionar esta característica no deseable en los estimadores de un modelo, se tasa en una primera etapa, el comportamiento de la variable endógena, el resultado de la prueba de inglés (Ingles_sb11) en función de los niveles de educación de los padres (Educa_padre, Educa_madre) tomados como instrumentos de los HLM, de acuerdo con la especificación (3).

$$
\text { ingles_sb11 } \mathrm{pro}_{\mathrm{i}, \mathrm{j}}=\gamma_{0}+\gamma_{1} \text { Educa_madre }_{\mathrm{i}, \mathrm{j}}+\mathrm{e}_{\mathrm{ij}}
$$

Se tomará como variable dependiente el resultado de inglés de la prueba Saber Pro ${ }^{6}$ (Ingles_sbpro) e incluyendo las covariables de la tabla 5 y la variable estimada ingles $s b 11_{\mathrm{i}, \mathrm{j}}$ como resultado de la especificación (3), según la especificaciones no reducidas mostradas en (4). 
ingles_sbpro ${ }_{\mathrm{i}, \mathrm{j}}$

$$
\begin{aligned}
& =\beta_{0 \mathrm{j}}+\beta_{1 \mathrm{j}} \text { ingles_sb } 11_{\mathrm{i}, \mathrm{j}}+\beta_{2 \mathrm{j}} \text { male }_{\mathrm{i}, \mathrm{j}} \\
& +\beta_{3 \mathrm{j}} \text { estuestrato }_{\mathrm{i}, \mathrm{j}}+\beta_{4 \mathrm{j}} \text { ingfamilia }_{\mathrm{i}, \mathrm{j}}+\beta_{5 \mathrm{j}} \text { trabaja }_{\mathrm{i}, \mathrm{j}}+\mathrm{u}_{\mathrm{ij}} \\
& \beta_{0, j}=\alpha_{0,0}+\alpha_{0,1} \text { privada }_{j}+\alpha_{0,2} \text { select }_{j}+\alpha_{0,3} \text { nmtc }_{j}+\alpha_{0,4} \text { nprtc }_{j}+v_{0, j} \\
& \beta_{1, j}=\alpha_{1,0}+\alpha_{1,1} \text { privada }_{j}+\alpha_{1,2} \text { select }_{j}+\alpha_{1,3} \text { nmtc }_{j}+\alpha_{1,4} \text { nprtc }_{j}+v_{1, j} \\
& \beta_{2, j}=\alpha_{2,0}+\alpha_{2,1} \text { privada }_{j}+\alpha_{2,2} \text { select }_{j}+\alpha_{2,3} \text { nmtc }_{j}+\alpha_{2,4} \text { nprtc }_{j}+v_{2, j} \\
& \beta_{3, j}=\alpha_{3,0}+\alpha_{3,1} \text { privada }_{j}+\alpha_{3,2} \text { select }_{j}+\alpha_{3,3} \text { nmtc }_{j}+\alpha_{3,4} \text { nprtc }_{j}+v_{3, j} \\
& \beta_{4, j}=\alpha_{4,0}+\alpha_{4,1} \text { privada }_{j}+\alpha_{4,2} \text { select }_{j}+\alpha_{4,3} \text { nmtc }_{j}+\alpha_{4,4} \text { nprtc }_{j}+v_{4, j} \\
& \beta_{5, \mathrm{j}}=\alpha_{5,0}+\alpha_{5,1} \text { privada }_{\mathrm{j}}+\alpha_{5,2} \text { select }_{\mathrm{j}}+\alpha_{5,3} \mathrm{nmtc}_{\mathrm{j}}+\alpha_{5,4} \mathrm{nprtc}_{\mathrm{j}}+\mathrm{v}_{5, \mathrm{j}}
\end{aligned}
$$

A partir de los resultados del modelo (4) es posible construir un índice de valor agregado para

\section{Resultados del modelo}

Elmodeloestimado segúnlaespecificación (4) da como resultado un parámetro que sirve para calcular el valor agregado de todas las instituciones discriminado por cada área de conocimiento de los programas académicos, cada institución según la especificación de la ecuación (1), el cual se analizará en la próxima sección.

\section{Gráfica 7}

Valor agregado de las universidades en la enseñanza de inglés discriminado por áreas de conocimiento de los programas académicos, 2002-2012

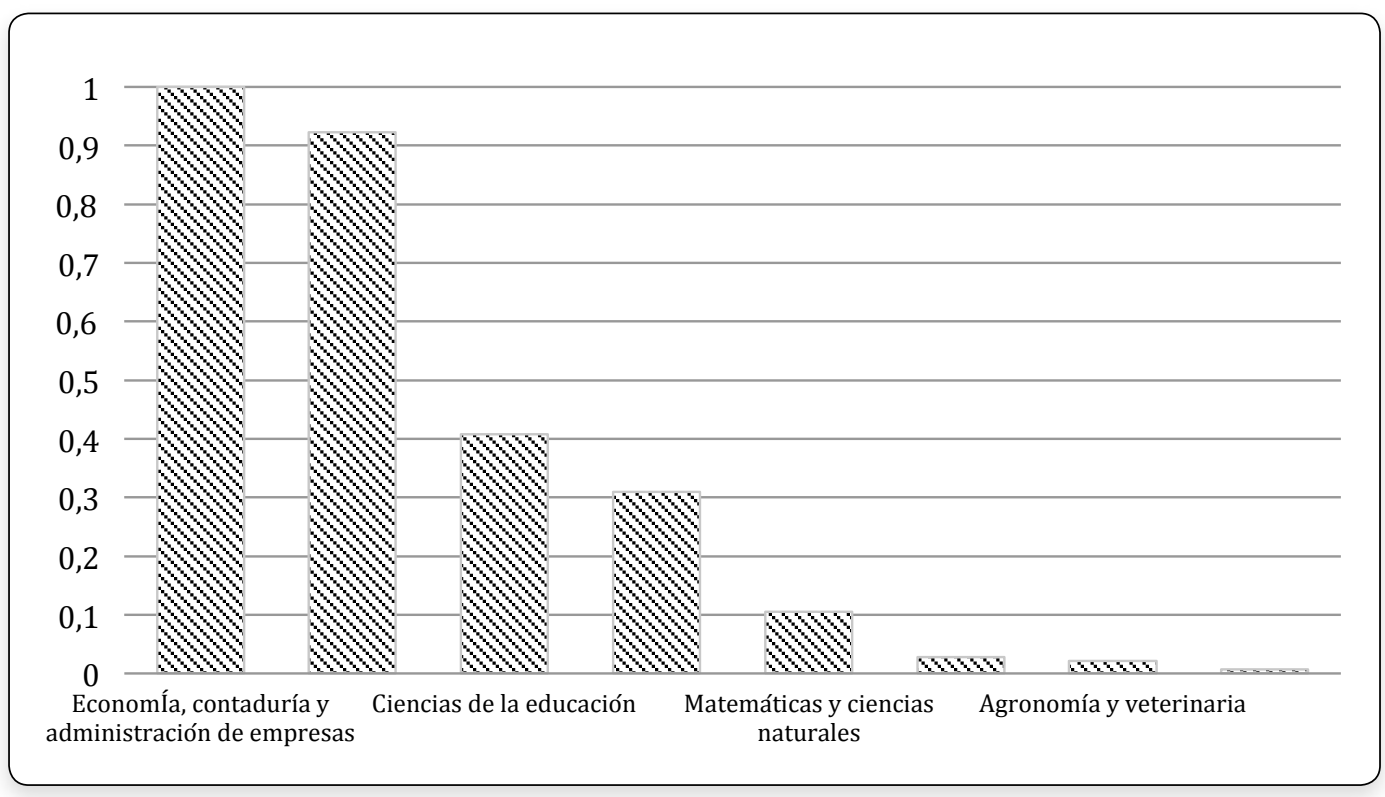

Fuente: elaboración propia con base en información del ICFES, 2015 
Tal como revela la gráfica 7 , los programas académicos de formación en pregrado en Colombia que más valor agregado aportan a la enseñanza del idioma inglés son aquellos que corresponden a los de economía, contaduría pública, administración de empresas y programas afines como negocios internacionales, comercio exterior y finanzas internacionales. Es posible que el enfoque internacional de estos programas académicos, determine su mayor aporte añadido o valor agregado en la enseñanza del idioma inglés. Vale la pena destacar que estos programas corresponden a un área de conocimiento, que es una de las demandadas (véase tabla 4). Según el modelo estimado las áreas que menos aportan son las ciencias sociales y humanas, agronomía y veterinaria y bellas artes.

Como en Colombia aún no es posible calcular el valor agregado de los niveles anteriores a la formación de pregrado universitario; no se puede medir el efecto escuela de la educación superior en comparación con los niveles precedentes.

\section{Conclusiones}

El PNB 2004-2019 estableció como algunas de sus metas que los estudiantes egresados de la educación media alcanzaran el nivel intermedio $\mathrm{B} 1 \mathrm{y}$ los que terminen la educación superior el B2, hacia el 2019. Los resultados de evaluaciones internas e internacionales reflejan el bajo desempeño de los estudiantes colombianos. En efecto, aunque Colombia tiene cada vez mayores puntajes en la prueba EPI practicada por EF en el periodo 2011-2014, la posición del país no mejora internacionalmente en el ámbito regional.

En el escenario doméstico, en el 2014 el $19 \%$ de los estudiantes que terminaron la educación media tenía un nivel igual o superior a B1 y en el 2013 el $10 \%$ de los estudiantes que concluyeron la educación superior de pregrado universitario tan solo superó el nivel B1. Como se observa, el estado actual del desempeño de los estudiantes colombianos se encuentra lejos de las metas planteadas en el PNB hacia el 2019, teniendo en cuenta que faltan cuatro años para llegar al año final proyectado por el programa. Las tendencias de los avances desde el 2007 muestran que las metas no serán alcanzadas y es necesario una reconsideración de los objetivos propuestos y un fortalecimiento agresivo de las estrategias y políticas de bilingüismo en el país en todos los niveles educativos.

Como en el país aún no se encuentran disponibles datos para medir el valor agregado de la educación básica y media, no es posible comparar los resultados del modelo estimado en el presente documento para la educación superior con los niveles educativos precedentes. Este debería ser un tema pendiente por analizar en futuras investigaciones a medida que el Icfes divulgue los resultados de las pruebas Saber 3, 5 y 9 con estructuras tipo panel de datos.

A partir de los análisis estadísticos se concluiría que un estudiante con un débil desempeño en la educación preuniversitaria en competencias en inglés, tendría un bajo resultado en la educación superior, así lo evidencia la fuerte correlación directa de ambas pruebas equivalente a 0,78. En este sentido es necesario reestructurar la formación universitaria en una segunda lengua para que realmente el valor agregado sea notable.

El resultado del modelo muestra que los programas académicos de economía, contaduría pública, administración de empresas, negocios internacionales, comercio exterior y finanzas internacionales son los que más valor agregado dan a la formación en inglés a sus estudiantes, en contraste con los programas de agronomía, veterinaria y bellas artes, cuya contribución es exigua comparada con los primeros. El valor agregado en la formación en inglés de los programas de ciencias de la educación representa el $40 \%$ del aporte de los primeros, evidenciando que es menester fortalecer la estructura curricular en segunda lengua de los futuros profesores. 


\section{Notas}

1 Artículo 21 de la ley 115 de 1994.

2 Considera tres niveles de clasificación: usuario básico: A1 y A2, usuario intermedio: B1 y B2 y usuario avanzado: $\mathrm{C} 1$ y $\mathrm{C} 2$.

3 El Icfes dispone de una base de acceso público con esta información pero con datos para 74.051 estudiantes, cantidad que asciende a menos de la mitad de la construida en el proyecto ejecutado.

${ }^{4}$ El test TOEFL (Test Of English as Foreign Language) es el examen de inglés más respetado en todo el mundo, reconocido por más de 9000 centros de estudios, universidades y organizaciones de más de 130 países.

5 Véase comentario en pie de página 6 de este documento.

6 A partir del año 2010, la prueba Saber Pro cambió su estructura evaluativa. Sin embargo el módulo de inglés se mantuvo dentro de las competencias genéricas evaluadas.

\section{Referencias}

Aitkin, M., \& Longford, N. (1986). Statistical modelling issues in school effectiveness studies. Journal of the Royal Statistical Society. Series A, 149, 1-43.

Benhabib, J., \& Spiegel, M. (1994). The role of human capital in Economic development: Evidence from aggregate cross - country data. Journal of Monetary Economics, 34(2), 143-174. doi: 10.1016/03043932(94)90047-7.

Birdsall, N., Ross, D. \& Sabot, R. (1997). Education, Growth and Inequality. In N. Birdsall y F. Jaspersen (Eds.), Pathways to Growth (pp. 93-127). Washington, D.C.: Inter-American Development Bank.
Bleakey, H., \& Chin, A. (2004). Language skills and earnings: evidence for childhood immigrants. The Review of Economics and Statistics, 86(2), 481496. doi:10.1162/003465304323031067.

Bogoya, J. D., \& Bogoya, J. M. (2013). An academic value-added mathematical model for higher education in Colombia. Ingeniería e Investigación. 33(2), 7681. Recuperado de http://www.revistas. unal.edu.co/index.php/ingeinv/rt/ printerFriendly/39521/42363

Bryk,A., \& Raudenbush, S.(1992). Hierarchical Linear Models: Applications and Data Analysis Methods. Newbury Park, C.A.: Sage Publications.

Chiswick, B. (1998). Hebrew Language Use. Journal of Population Economics, 11(2), 253-71.

Chiswick, B., Patrinos, A., \& Hurst, M. (2000). Indigenous Language Skills and the Labor Market in a Developing Economy: Bolivia. Economic Development and Cultural Change, 48(2), 347-67. doi: doi:10.1086/452462.

Courgeau, D. (2007). Multilevel synthesis. Dordrecht: Springer - Verlag.

Crystal, D. (2003). English as a global language. Cambridge: Cambridge University Press.

Draper, D. (1995). Inference and hierarchical modeling in social sciences. Journal of Educational and Behavioral Statistics, 20(2), 115-149.

Education First. (2014). English Proficiency Index. Recuperado de http://www.ef.com. co/epi/

Fandiño, Y., Bermúdez, J., \& Lugo, V. (2012). Retos del Programa Nacional de Bilin- 
güismo. Colombia Bilingüe. Educ. 15(3), 363-381.

Fondo Monetario Internacional (2016). World Economic Outlook Database. Recuperado de http://www.imf.org/external/pubs/ft/ weo/2016/01/weodata/index.aspx

Goldstein, H. (2001). Using pupil performance data for judging school and teachers: scope and limitations. British Educational Research Journal, 27(4), 433-442.

Goldstein, H., Huiqi, P., Rtah, T., \& Hill, N. (2000). The use of value-added information in judging school performance. Perspectives on Education Policy. Londres: Institute of Education; University of London.

Goldstein, H., \& Spiegelhalter, D. (1996). League tables and their limitations: statistical issues in comparison of institutional performance. Journal of the Royal Statistical Society. Series A, 159(3), 385-443.

Graddol, D. (2006). English Next. Londres: British Council.

Hall, R., \& Jones, C. (1999). Why do some countries produce so much more output per worker tan others?. Quarterly Journal of Economics, 114(1), 83-116.

Hanushek, E., Kain, J., Rivkin, S., \& Branch, G. (2005). Charter School Quality and Parental Decision Making with School Choice (National Bureau of Economic Research, Workinkg paper No. 11252). Cambridge. Recuperado de http://www. nber.org/papers/w11252.pdf

Helliwell, J. (1999). Language and Trade. En A. Breton (Ed.). Exploring the economics of Language (pp. 5-30). Canadá: Canada Heritage.
Instituto Colombiano para la Evaluación de la Educación -[ICFES]. (2015). Bases de datos. Recuperado de: http://www. icfes.gov.co/index.php/investigadoresposgrado/acceso-a-bases-de-datos.

Instituto Nacional de Estadística e Informática de Perú -[INEI]. (2014). Indicadores de educación por departamentos. Recuperado de: https://www.inei.gob.pe/media/ MenuRecursivo/publicaciones_digitales/ Est/Lib1293/index.html.

Keller, W. (2002). Geographical Localization of International Technology Diffusion. American Economic Review, 92(1), 120 - 142.

Lee, C. G. (2009). English language and economic growth: Cross country empirical evidence. Journal of Economic and Social Studies, 2(1), 5-20.

Ley 115 de 1994. Por la cual se expide la ley general de educación. Diario Oficial No. 41.214. Congreso de la República de Colombia, febrero de 1994

Lindley, D., y Smith, A. (1972). Bayes estimates for the linear model. Journal of the Royal Statistical Society. Series B, 34(1), 1-41.

Longford, N.T. (1993). Random Coefficient models. Oxford. Clarendon Press.

Lucas, R. (1989). On the Mechanics of Economic Development. Journal of Monetary Economics, 22, 3-42.

Mankiw, N., Romer, D., \& Weil, D. (1992). A contribution to the empirics of economic growth. Quarterly Journal of Economics, 107(2), 407-437.

Manzi, J., San Martin, E., \& Van Bellegem, S. (2014). School system evaluation by value added analysis under endogeneity. Psychometrika, 79(1), 130-153. 
Marschan, R., Welch, D., \& Welch, L. (1997). Language: The Forgotten Factor in Multinational Management. European Management Journal, 15(5), 591-98. doi: 10.1016/S0263-2373(97)00038-8.

Mcfrey, D., Lockwood, J., Koretz, D., Louis, T., \& Hamilton, L. (2004). Models for Value-Added Modeling of Teacher Effects. Journal of Education and Behavoir Statistics, 29(1), 67-101. doi: 10.3102/10769986029001067.

McMahon, W. (1999). Education and Development. Oxford: Oxford University Press.

Meghir, C., \& Rivkin, S. (2011). Econometric Methods for research in Education. Handbook of the Economics of Education, 3, 1-87.

Meyer, R. (1997). Value-Added Indicators of School Performance: A Primer. Economics of Education Review, 16(3), 283-301.

Ministerio de Educación Nacional - [MEN]. (2006). Estándares básicos en competencias en lengua extranjera. Recuperado de: http://www.colombiaaprende.edu.co/ $\mathrm{html} /$ mediateca/1607/articles-115375_archivo.pdf

Organización para la Cooperación y el Desarrollo Económico -[OECD]. (2013). Assessment of Higher Education Learning Outcomes AHELO (Feasibility Study Report. Vol. 2- Data Analysis and National Experiences). Recuperado de http://www. oecd.org/education/skills-beyond-school/ AHELOFSReportVolume2.pdf

Pecorari, D., Shaw, P, Malsmtrom, H., \& Irvine, A. (2011). Teachers of english to speakers of other languages. TESOL Quarterly, 45(2), 313-333.

Rabe-Hesketh, S., \& Skrondal, A. (2012). Multilevel and Longitudinal Modeling using Stata (Vol. 1, 3a ed.) Texas: College Station.

Raudenbush, S. (2004). What Are Value-Added Models Estimating and What Does This Imply for Statistical Practice?. Journal of Educational and Behavioral Statistics, 29(1), 121-129.

Raudenbush, S., \& Bryk,A. (2002). Hierarchical Linear Models: Applications and Data Analysis Methods (2a ed.). Thousand Oaks, CA: Sage Publications.

Raudenbush, S., \& Willms J. (1995). The estimation of school effects. Journal of Educational and Behavioral Statistics, 20(4), 307-335.

Romer, P. (1990). Endogenous technological change. Journal of Political Economy, 99(5), 71-102.

San Martin, E., \& Carrasco, A. (2012). Clasificación de escuelas en la nueva institucionalidad educativa: contribución de modelos de valor agregado para una responsabilización justa. Centro de Políticas Públicas Universidad Católica de Chile, 7(53), 1-16 Recuperado de http://politicaspublicas.uc.cl/wp-content/uploads/2015/02/ descargar-clasificacion-de-escuelas-enla-nueva-institucionalidad-educativa.pdf

Sanders, W. (2000). Value Added Assessment from Student Achievement Data, Opportunities and Hurdles. Journal of Personnel Evaluation in Education, 14(4), 329339.

Smith A. (1973). A general Bayesian linear model. Journal of the Royal Statistical Society. Series B, 35(1), 67-75. 
